\title{
La franc-maçonnerie comme exotisme dans «Guerre et Paix »
}

Julie Bouvard

\section{(2) OpenEdition}

1 Journals

Édition électronique

URL : http://journals.openedition.org/edl/377

DOI : $10.4000 /$ edl. 377

ISSN : 2296-5084

Éditeur

Université de Lausanne

\section{Édition imprimée}

Date de publication : 15 septembre 2009

Pagination : 59-68

ISBN : 978-2-940331-20-8

ISSN : 0014-2026

Référence électronique

Julie Bouvard, «La franc-maçonnerie comme exotisme dans " Guerre et Paix » », Études de lettres [En ligne], 2-3 | 2009, mis en ligne le 15 septembre 2012, consulté le 19 décembre 2020. URL : http:// journals.openedition.org/edl/377 ; DOI : https://doi.org/10.4000/edl.377 


\section{LA FRANC-MAÇONNERIE COMME EXOTISME DANS "GUERRE ET PAIX"}

Interprétant les errements du héros comme un voyage vers un inconnu lointain, l'article propose une analyse serrée des épisodes maçonniques du roman de Tolstoï. Celle-ci permet de montrer que cette quête spirituelle, décisive pour la découverte de sa propre différence, ne peut aboutir sans un retour vers le réel et vers l'Autre.

Si l'on se réfère au Grand Larousse Encyclopédique, l'exotisme désigne d'abord «le caractère d'une ouvre qui évoque les mœurs ou les paysages étrangers", puis "l'ensemble des êtres et des choses ayant ce même caractère», enfin, "le goût des choses exotiques " ${ }^{1}$. Plus loin, il nous est dit que si le terme s'introduit en Europe au cours du XIX siècle pour signifier le goût des formes d'art et des mœurs des peuples lointains, il apparaît dès la découverte du Nouveau Monde, dont le choc stimule les imaginations et révolutionne les esprits: dès lors, l'artiste et le penseur occidentaux posent un autre regard sur la société dite civilisée. A cet égard, on peut parler d'un exotisme "moral», dont les plus célèbres illustrations - le cannibale naïf de Montaigne, le bon sauvage de Rousseau, les Lettres persanes de Montesquieu - révèlent le principe: l'altérité de l'Etranger, renforcée par une différenciation marquée de ses signes extérieurs, renvoie à soi le reflet inversé de ses propres tares et devient l'incarnation de ses propres idéaux. Partant de ces définitions, nous aimerions montrer comment le rôle joué par la franc-maçonnerie dans la quête spirituelle de Pierre Bezoukhov permet de l'apparenter à une forme particulière d'exotisme que nous appellerons «spirituel», et qui se traduit par une

I. Grand Larousse encyclopédique, IV. 
appropriation de la différence de l'Autre pour parvenir à une réconciliation avec soi-même. Trois thématiques, intrinsèquement liées, serviront d'appui à notre développement: la quête spirituelle présentée comme un voyage; le rituel initiatique permettant l'assimilation de signes extérieurs étrangers; enfin, la fascination qu'exerce sur le profane la découverte d'un milieu clos, régi par des codes qui lui échappent et dont le mystère renforce l'attrait.

Au chapitre I de la deuxième partie du tome II de Guerre et Paix, Bezoukhov, après son duel avec Dolokhov, traverse une crise morale qui l'oblige à reconsidérer la vie qu'il a menée jusqu’alors :

"Qu'est-ce qui est mal? Qu'est-ce qui est bien? Que faut-il aimer, que faut-il haïr? Pour quoi vivre et que suis-je? Qu'est-ce que la vie, qu'estce que la mort? Quelle est la force qui régit tout?» se demandait-il. Et il n'y avait de réponse à aucune de ces questions, sauf une réponse illogique, qui ne répondait pas du tout à ces questions. Cette réponse était: «Tu mourras et tout sera fini. Tu mourras et tu sauras tout, ou tu cesseras de poser des questions.» ${ }^{2}$

Il est remarquable que ce point de rupture métaphysique s'accompagne d'un éloignement physique. Ce monologue intérieur intervient, en effet, lorsque le personnage a physiquement rompu avec son ancien mode d'existence: il a quitté sa femme, il a quitté Moscou et passe la nuit dans un relais sur la route de Pétersbourg. Un relais, c'est-à-dire, littéralement, un lieu de passage où l'on se trouve privé de ses repères habituels, exposé à l'inconnu. En outre, l'idée de passage suppose que d'un lieu ou d'un état initial, on passe à un autre: c'est bien dans ce relais que Bezoukhov fait la rencontre déterminante du franc-maçon Bazdeev, qui lui ouvrira les portes, au sens propre comme au figuré, d'un monde nouveau. Au demeurant, la métaphore du voyage sera clairement explicitée à la fin du chapitre IV qui clôt le rituel d'intégration de Bezoukhov au sein de la loge de Pétersbourg:

La séance était terminée, et en rentrant chez lui, Pierre avait l'impression d'être revenu de quelque lointain voyage qui avait duré des dizaines d'années, au cours duquel il s'était complètement transformé et avait rompu avec son ancien genre de vie et ses anciennes habitudes ${ }^{3}$.

2. L. Tolstoï, Guerre et Paix, II, p. 90.

3. Ibid., p. 112. 
L'association entre le chemin spirituel et le voyage, plus précisément la navigation, est sans doute un des lieux communs les plus anciens et les plus féconds de l'imagerie chrétienne primitive. Un autre, consubstantiel, est la vision du paradis terrestre en tant qu'île, située quelque part sur terre bien que ne figurant sur aucune carte: cette vision, comme on le sait, représente également le principal symbole de la pensée utopique. Cette même métaphore du cheminement spirituel constitue une récurrence dans l'œuvre de Tolstoï, ainsi que l'illustre la célèbre parabole de sa Confession:

Il m'était arrivé quelque chose comme ceci : à un moment dont je ne me souvenais plus, on m'avait fait monter dans une barque, on m'avait poussé sur l'eau à partir d'une rive inconnue, on m'avait montré ma direction - la rive opposée - puis, glissant les rames entre mes mains inexpérimentées, on m’avait laissé tout seul. [...] Parvenu au milieu du fleuve, serré entre les barques et les navires emportés par le courant, j'avais fini par perdre complètement le sens du mouvement, j'avais jeté les rames. Partout autour de moi, des voyageurs précipités par la force des rames ou des voiles poussaient des clameurs de joie et de jubilation, m'assurant et s'assurant les uns les autres qu'il ne pouvait y avoir d'autre direction. Je les avais crus et je m'étais joint à eux ${ }^{4}$.

Cette aventure, cette plongée dans l'inconnu que représente la quête de soi, paraît, dans un premier temps, nécessiter des guides, tout du moins des compagnons: c'est d'une communauté qu'il s'agit ici, qui, au niveau symbolique, devient l'incarnation physique, voire géographique, d'un paradis terrestre, véritable ou-topos, inscrit sur aucune carte et néanmoins là, immédiatement présent. De même, la franc-maçonnerie, à laquelle Tolstoï, sans jamais y avoir adhéré, s'est cependant intéressé de près, devient, pour un temps, au regard de son héros, une Terre Promise: n'étant ni une religion, puisque toutes y sont tolérées, ni un parti, puisque nulle idéologie n'y est revendiquée, elle affirme constituer une communauté dont tous les membres, unis dans l'accomplissement spirituel, sont frères, nonobstant les fonctions et positions sociales de chacun.

Mais la seule métaphore du voyage ne nous permettrait pas de parler d'exotisme; pour ce faire, il faut qu'elle s'accompagne de la notion d'étrangeté. Et c'est bien d'étrangeté, au sens de tchoujdost', d'altérité

4. L. Tolstoï, Confession. 
incompatible, qu'il est d'abord question dans le dialogue entre Pierre Bezoukhov et Bazdeev au relais de Torjok:

Je crains, dit Pierre en souriant, partagé entre la confiance que lui inspirait le franc-maçon et l'habitude de railler les croyances maçonniques, je crains d'être loin [en russe dalek] de comprendre... comment vous dire, je crains que ma conception de l'univers ne soit trop opposée à la vôtre pour que nous puissions nous comprendres.

Ce "dalek», dans le contexte qui nous préoccupe, est éloquent; initialement, Pierre se sent aux antipodes de la pensée franc-maçonne, dont la société représente à ses yeux un lointain, au sens fort du terme: lui étant étrangère en tout (origine, organisation, principes, philosophie, etc.), elle lui est, de fait, inaccessible. A lui seul, le terme dalek traduit ici l'impossibilité de l'échange qui caractérise une altérité trop radicale et que Bazdeev, dans sa réponse, s'appliquera à effacer:

"Contemple ton être intérieur avec les yeux de l'âme et demande-toi si tu es content de toi. A quoi es-tu arrivé en te laissant guider par ta seule raison? Qu'es-tu? Vous êtes jeune, vous êtes riche, vous êtes intelligent, instruit, monsieur. Qu'avez-vous fait de tous ces biens qui vous ont été dévolus? Etes-vous satisfait de vous-même et de votre vie?»/ - Non, je hais ma vie, fit Pierre en se rembrunissant ${ }^{6}$.

Dans un discours où il fait adroitement alterner le "vous» de politesse et le «tu» de l'emphase, Bazdeev énonce à Bezoukhov ses propres interrogations, ses propres doutes, en reprenant quasiment ses propres termes, pour lui faire entrevoir la possibilité d'une issue, à proprement parler d'une résurrection, entraînant une adhésion de Pierre d'autant plus complète qu'elle semblait, à l'origine, inconcevable: ses fréquents «Oui, oui, c'est ainsi!» qui interrompent, en les ponctuant, les propos du franc-maçon, traduisent bien l'aspect émotionnel de cette adhésion, suscitée avant tout par l'émerveillement de se reconnaître en l'autre. Ici, nous touchons à un point qui nous paraît essentiel, parce que spécifiquement russe, du moins en comparaison de la pensée occidentale. Contrairement, en effet, aux philosophes et esthètes européens pour lesquels l'altérité du «bon sauvage» paraît d'autant plus attirante qu'elle

5. L. Tolstoï, Guerre et Paix, II, p. 94. C'est nous qui soulignons.

6. Ibid., p. 97. 
est considérée (et nous insistons sur le terme) comme absolue, pour le Russe, l'étrangeté de l'Autre est exotique, à savoir désirable, parce que le soi s'y reconnaît. Somme toute, l'altérité du «bon sauvage» n'est que purement formelle, voire stylistique, puisque son véritable but est de servir de faire-valoir à une morale strictement nationale. Chez Tolstoï, en revanche, la reconnaissance de soi dans l'Autre n'en nie pas l'altérité mais, au contraire, en souligne la différence, dans une sorte de fascination émerveillée. L'Autre serait en quelque sorte un travestissement de Soi dont il reflèterait l'image idéale, laissant entrevoir la possibilité d'un perfectionnement. Toutefois, cette reconnaissance renvoie à une acceptation de la différence qui relève plus du fantasme que de la réalité. Ainsi, dès cette première et fortuite rencontre avec Bazdeev, le mécanisme de l'enthousiasme de Bezoukhov est clairement énoncé:

Pierre, le cœur défaillant, regardait le franc-maçon avec des yeux brillants, l'écoutait sans l'interrompre, sans lui poser de questions, mais croyait de toute son âme à ce que lui disait cet inconnu. Se laissait-il convaincre par la logique des arguments du franc-maçon ou, comme les enfants, par ses intonations, son accent persuasif et cordial, le tremblement de sa voix qui, par moments, lui coupait presque la parole [...]; quoiqu'il en fût, il désirait croire de toute son âme, et il croyait, il éprouvait une sensation joyeuse d'apaisement, de rénovation et de retour à la vie?

Nous retrouvons ici le besoin de projeter sur l'extérieur un idéal essentiellement intérieur, c'est-à-dire sien, tel que ce besoin est décrit dans Confession, l'Autre acquérant de fait une dimension d'idéal fantasmatique, laquelle, précisément, caractérise toutes les formes d'exotisme.

Mais pour parvenir à cet accomplissement du soi tant désiré, il faut à son tour se travestir, littéralement "changer de peau", ainsi que nous le suggère la description du rituel d'initiation de Bezoukhov dans la loge pétersbourgeoise. Cette description occupant deux chapitres entiers, il serait fastidieux d'en proposer une analyse détaillée; nous nous contenterons d'en souligner certains moments significatifs. Pour commencer, rappelons que, ainsi que le veut le rituel, Pierre est amené au lieu de son initiation les yeux bandés: sa perception de l'espace où il évolue et des événements qu'il est en train de vivre est par conséquent purement

7. Ibid., p. 96. C'est nous qui soulignons. 
sensitive, ce qui renforce le parallèle avec un étranger se retrouvant en terre inconnue. Présenté ainsi, l'espace spirituel qu'est la loge maçonnique devient un lieu à part entière, possédant une géographie propre, dont il faut assimiler les repères. Là encore, la notion d'inconnu et la supériorité qui lui est attribuée découlent plus d'un désir que d'un constat objectif:

Au moment où la porte s'était ouverte et où l'inconnu était entré, Pierre avait été envahi par un sentiment de crainte et de respect semblable à celui que, dans son enfance, il éprouvait à confesse; il se trouvait en tête à tête avec un homme qui lui était parfaitement étranger dans la vie de tous les jours et proche par la fraternité des hommes. [...] S'étant approché, [Pierre] reconnut dans l'orateur une personne de connaissance, un certain Smolianinov, mais il lui répugnait de penser qu'il connaissait le nouveau venu: ce n'était qu'un frère et un vertueux instructeur ${ }^{8}$.

Par ailleurs, plus les ordres qu'on lui donne lui semblent étranges, plus vif est son empressement à les exécuter: tout au long du processus, Pierre n'aura cesse de prendre les symboles rituels au pied de la lettre, prêt, s'il le faut, à s'empaler sur les épées pointées sur sa poitrine. Outre l'aspect évidemment parodique de cet épisode, on peut y voir un déplacement significatif des symboles en question, qui, de simples codes rituels, deviennent les signes extérieurs de la supériorité de l'Autre, détenteur d'une vérité aussi tangible et immuable que ces signes eux-mêmes:

Espérant commencer une vie entièrement nouvelle, entièrement différente de l'ancienne, il s'attendait à des choses extraordinaires, plus extraordinaires encore que ce qu'il voyait. La tête de mort, le cercueil, l'Evangile, il lui semblait s'être attendu à tout cela, s'être attendu à plus encore 9 .

Ce n'est donc pas tant l'origine historique de la franc-maçonnerie qui en fait l'exotisme, mais sa dimension spirituelle et philosophique, comme en témoigne l'échec même de l'expérience franc-maçonne de Bezoukhov. Celui-ci survient après le retour de Pierre où il aura

8. Ibid., p. 103. C'est nous qui soulignons.

9. Ibid., p. 102. 
fréquenté les personnalités les plus influentes de la franc-maçonnerie et, comme il nous est dit, où "il avait été initié à bien des mystères» ${ }^{10}$. Plus Bezoukhov comprend les textes, plus se ternit l'aura de supériorité spirituelle que détenaient, à ses yeux, ses compagnons francs-maçons: la figure idéalisée du frère spirituel, pur et juste, que Bezoukhov s'est plu à esquisser, s'estompe derrière la véritable personne, dont il connaît le nom, le passé, le présent, les ambitions et les vices. Profondément déçu, Pierre se détourne alors de la société franc-maçonne qu'il accuse de se dévoyer au profit d'intrigues politiques. Néanmoins, le rêve de trouver son idéal ne s'arrêtera pas à cet échec et, là encore, c'est au sein d'une nouvelle communauté qu'il pensera le réaliser, la communauté paysanne que représentent les soldats qu'il va fréquenter au front:

«Dieu soit loué, tout cela est fini, se dit Pierre en se recouvrant de nouveau la tête. Oh! quelle chose horrible que la peur et combien honteusement je m'y suis laissé aller! Et eux tout le temps, jusqu'au bout, ils ont été fermes, calmes...»Eux [oni en russe] étaient pour lui les soldats, ceux de la batterie, et ceux qui lui avaient donné à manger, et ceux qui priaient devant l'icône. Eux, ces hommes étranges qui jusqu'alors lui étaient inconnus, se détachaient nettement dans sa pensée et tranchaient sur tous les autres hommes ${ }^{11}$.

Ce "oni", souligné par Tolstoï lui-même, est révélateur. De nouveau, en effet, l'Autre devient l'idéal à atteindre dans la mesure où il ne se réduit pas à l'individu dont la solitude reflète l'imperfection, mais représente un Tout solidaire. C'est ce Tout surpuissant, opposé à la faiblesse, désemparé du Un, que Pierre cherche à intégrer, en essayant de transcender le «je» de la solitude, du doute, de l'angoisse et du désir dans un «nous» stable, ferme, clos, et obéissant à des règles rassurantes par leur immuabilité. La nature occidentale de Pierre, nourrie des Lumières et convaincue de la suprématie de la raison, ayant été déçue, c'est à sa «nature russe» qu'il va désormais faire appel, usant exactement des mêmes procédés et poursuivant les mêmes buts. Du reste, le parallèle entre les deux communautés est clairement énoncé dans le texte:

Io. Ibid., p. 220.

II. Ibid., p. 366. C'est nous qui soulignons en gras, l'italique est de Tolstoï. 
Et dans l'imagination [de Pierre] défilèrent le dîner au club, au cours duquel il avait provoqué Dolokhov, et son bienfaiteur à Torjok. Et voici qu'il se représente une tenue solennelle de la loge. Cette séance a lieu au Club anglais. Et quelqu'un de familier, de proche, de cher est assis au bout de la table; Mais c'est lui! c'est le bienfaiteur. [...] Et eux, de toutes parts, avec leurs visages simples, bons, fermes, entouraient le bienfaiteur ${ }^{12}$.

Là encore, le désir d'acquérir les vertus de l'Autre s'accomplira dans l'adoption de ses signes distinctifs extérieurs, adoption qui, symboliquement, représente la transcendance tant rêvée de l'individualité imparfaite en un collectif tout-puissant:

«Ecoute, ajouta [Pierre] en prenant Guerassime par le bouton de sa redingote [...]. Ecoute, tu sais qu'il va y avoir une bataille demain?... / On le dit, répondit Guerassime. / Je te demande de ne dire à personne qui je suis. Et fais ce que je vais te demander... / A vos ordres, dit Guerassime. Faut-il vous servir à manger? / Non, mais il me faut autre chose. J'ai besoin de vêtements de paysan et d'un pistolet, dit Pierre qui rougit brusquement. / A vos ordres", répéta Guerassime, après un instant de réflexion ${ }^{13}$.

Les vêtements de paysan et le pistolet du soldat vont jouer le même rôle que le tablier blanc et l'épée du franc-maçon, à savoir celui de médiateur magique entre ce que l'on est et ce que l'on veut être, entre l'extérieur et l'intérieur: "L'habit fera le moine» semble affirmer ce besoin quasi compulsif de travestissement. Par ailleurs, il rappelle toute la tradition du travestissement exotique, lorsque le Blanc se pare des plumes du Nouveau Monde, du masque africain, ou encore du peignoir oriental. A cet égard, l'exemple russe le plus éloquent de l'exil dans une communauté considérée comme exotique pour fuir les vices de la civilisation et s'en purifier est évidemment le poème Les Tsiganes de Pouchkine. Ici aussi, cette aspiration se solde par un échec, non pas imputable à la communauté d'accueil mais à la faiblesse de l'étranger qui l'a intégrée: ce n'est pas Aleko qui, déçu, quitte la communauté tsigane, c'est cette dernière qui le châtie pour son crime en le bannissant.

I2. Ibid., p. 367. C'est nous qui soulignons en gras, l'italique est de Tolstoï.

I3. Ibid., p. 406. C'est nous qui soulignons. 
"Chassez le naturel, il revient au galop" serait, en quelque sorte, la réponse pouchkinienne au fantasme exotique.

En ce qui concerne le héros de Tolstoï, il va falloir attendre le choc de l'emprisonnement - dans lequel, au niveau symbolique, on peut voir une autre image de l'exil - et la rencontre avec Karataïev pour que Pierre comprenne quau même titre que la paix à laquelle il aspire, le voyage qui y mène est solitaire et intérieur: Karataïev, ultime représentant de l'idéal spirituel de Bezoukhov, déraciné comme lui puisque arraché à sa communauté d'origine, n'apparaît cependant plus en tant que modèle à copier, infime partie reflétant la perfection d'un Tout, mais comme affirmation de sa propre singularité qui, seule, peut indiquer la direction à suivre. Au camp de prisonniers, les pieds nus de Bezoukhov, sa barbe et ses cheveux longs ne sont plus des emprunts artificiels, mais la conséquence de son mode de vie, tout comme la joie et la paix intérieures qu'il éprouve sont authentiquement ressenties, et non plus stimulées par l'imagination dans une vaine tentative de combler un désir toujours insatisfait. Le véritable but est donc non pas d'acquérir un Ailleurs qui, par définition, ne saurait jamais être qu'un Lointain, mais d'accepter sa propre différence. C'est précisément, semble-t-il, le rêve et l'échec de ce que nous avons appelé «l'exotisme spirituel» dans Guerre et Paix. Ils révèlent avec force que, pour l'accomplissement de soi, l'individu qui se cherche ne peut faire l'économie d'un détour par l'extérieur, dans la mesure où l'acceptation de sa singularité passe d'abord et avant tout par l'expérience de sa propre altérité dont l'Autre est à la fois le reflet et le dépassement.

Julie Bouvard

Lausanne 


\section{BIBLIOGRAPHIE}

Grand Larousse encyclopédique en dix volumes, t. IV, Paris, Larousse, 1961.

Tolstö̈, L., Guerre et Paix, t. II, traduit par Elizabeth Guertik, Lausanne, Editions Rencontre, 1961.

—, Confession, suivi de Quelle est ma foi? et de Pensées sur Dieu, traduit par Luba Jurgenson, Lausanne, Pygmalion, 1998. 